Technical Report

3 March 2010

\title{
Recursive three term recurrence relations for the Jacobi polynomials on a triangle
}

\author{
Shayne Waldron \\ Department of Mathematics, University of Auckland, Private Bag 92019, Auckland, New Zealand \\ e-mail: waldron@math.auckland.ac.nz (http:www.math.auckland.ac.nz/ waldron)
}

\begin{abstract}
Given a suitable weight on $\mathbb{R}^{d}$, there exist many (recursive) three term recurrence relations for the corresponding multivariate orthogonal polynomials. In principle, these can be obtained by calculating pseudoinverses of a sequence of matrices. Here we give an explicit recursive three term recurrence for the multivariate Jacobi polynomials on a simplex. This formula was obtained by seeking the best possible three term recurrence. It defines corresponding linear maps, which have the same symmetries as the spaces of Jacobi polynomials on which they are defined. The key idea behind this formula is that some Jacobi polynomials on a simplex can be viewed as univariate Jacobi polynomials, and for these the recurrence reduces to the univariate three term recurrence.
\end{abstract}

Key Words: (recursive) three term recurrence relations, multivariate Jacobi polynomials, Legendre polynomials on a triangle, barycentric coordinates, symmetry group,

AMS (MOS) Subject Classifications: primary 33C50, 33F05, 42C05 secondary 33C20, 33C65, 33C80, 41A10, 42C15, 


\section{Introduction}

A sequence of univariate orthogonal polynomials $\left(p_{n}\right)$ satisfies a three term recurrence

$$
p_{n+1}(x)=\left(A_{n} x+B_{n}\right) p_{n}(x)-C_{n} p_{n-1}(x), \quad n \geq 0, \quad p_{-1}:=0 .
$$

This can be viewed as a formula for the inclusion of vector spaces

$$
\mathcal{P}_{n+1} \subset \operatorname{span}\left(\Pi_{1} \mathcal{P}_{n}\right) \oplus_{a} \mathcal{P}_{n-1}, \quad \mathcal{P}_{n}:=\operatorname{span}\left\{p_{n}\right\}
$$

where $\oplus_{a}$ is an algebraic direct sum, i.e., $\operatorname{span}\left(\Pi_{1} \mathcal{P}_{n}\right) \cap \mathcal{P}_{n-1}=\{0\}$, and $\Pi_{k}=\Pi_{k}\left(\mathbb{R}^{d}\right)$ is the space polynomials of degree $\leq k$. In particular, the coefficients $A_{n}, B_{n}, C_{n}$ are unique.

For $\mathcal{P}_{n}$ the space of multivariate orthogonal polynomials of degree $n$ for some weight on $\mathbb{R}^{d}$ (details below) the situation is more complicated. Here

$$
\mathcal{P}_{n+1} \subset \operatorname{span}\left(\Pi_{1} \mathcal{P}_{n}\right)+\mathcal{P}_{n-1}
$$

but the above sum may not be direct. For example, the orthogonal polynomials on the triangle (with constant weight) satisfy

$$
\mathcal{P}_{3} \subset \operatorname{span}\left(\Pi_{1} \mathcal{P}_{2}\right)=\mathcal{P}_{1} \oplus \mathcal{P}_{2} \oplus \mathcal{P}_{3},
$$

with $\oplus$ the orthogonal direct sum, so that

$$
\operatorname{span}\left(\Pi_{1} \mathcal{P}_{2}\right) \cap \mathcal{P}_{1}=\mathcal{P}_{1}, \quad \operatorname{dim}\left(\mathcal{P}_{1}\right)=2 .
$$

Thus the cubic orthogonal polynomials can be expressed a linear combination of quadratic ones multiplied by linear polynomials, without needing the linear orthogonal polynomials! See Example 1 of Section 3 for the details. This is quite different from the univariate case where $C_{n}>0, n \geq 1$.

Since $\operatorname{span}\left(\Pi_{1} \mathcal{P}_{n}\right)$ is orthogonal to $\Pi_{n-2}$, we have the inclusion

$$
\operatorname{span}\left(\Pi_{1} \mathcal{P}_{n}\right) \subset \mathcal{P}_{n+1} \oplus \mathcal{P}_{n} \oplus \mathcal{P}_{n-1} \quad \text { (orthogonal direct sum) }
$$

This map, and formulas for it, are sometimes referred to as three term recurrence relations (cf [DX01]). A version of Favard's theorem holds for this map (see [K82a], [K82b], [X93]). However, it is not recursive in the sense that it allows orthogonal polynomials in $\mathcal{P}_{n+1}$ to be calculated from those of degrees $n$ and $n-1$ in a similar fashion to the univariate case. We will call any pair of linear maps (or the formulas defining them)

$$
B_{n}: \mathcal{P}_{n+1} \rightarrow \operatorname{span}\left(\Pi_{1} \mathcal{P}_{n}\right), \quad C_{n}: \mathcal{P}_{n+1} \rightarrow \mathcal{P}_{n-1}
$$

with

$$
f=B_{n}(f)+C_{n}(f), \quad \forall f \in \mathcal{P}_{n+1}
$$

a recursive three term recurrence. Such a recurrence is recursive, in the above sense, since an orthogonal polynomial $f \in \mathcal{P}_{n+1}$ is uniquely determined as the projection of its leading term (homogeneous term of degree $n+1$ ) onto $\mathcal{P}_{n+1}$. 
In principle, a recursive three term recurrence can be calculated using the inclusion map (1.1) as follows. Let $Q_{n}$ be the orthogonal projection onto $\mathcal{P}_{n}$, and

$$
L=\left.Q_{n+1}\right|_{\operatorname{span}\left(\Pi_{1} \mathcal{P}_{n}\right)}: \operatorname{span}\left(\Pi_{1} \mathcal{P}_{n}\right) \rightarrow \mathcal{P}_{n+1}
$$

be the orthogonal projection of $\operatorname{span}\left(\Pi_{1} \mathcal{P}_{n}\right)$ onto $\mathcal{P}_{n+1}$ (this is clearly onto by considering leading terms). Let $R$ be any right inverse of $L$, i.e., an injective linear map

$$
R: \mathcal{P}_{n+1} \rightarrow \operatorname{span}\left(\Pi_{1} \mathcal{P}_{n}\right)
$$

which preserves leading terms. Then $f-R f$ is a polynomial of degree $n$, which is orthogonal to $\Pi_{n-2}$, and so belongs to $\mathcal{P}_{n} \oplus \mathcal{P}_{n-1}$. Hence we obtain the recursive three term recurrence

$$
f=\left(R f+Q_{n}(f-R f)\right)+Q_{n-1}(f-R f) \in \operatorname{span}\left(\Pi_{1} \mathcal{P}_{n}\right)+\mathcal{P}_{n-1}, \quad \forall f \in \mathcal{P}_{n+1} .
$$

By taking $R$ to be the $B_{n}$ of (1.2), it follows that all recursive three term recurrences can be obtained in this way. One choice for a general weight (described via the monic orthogonal polynomials) and the associated evaluation algorithms is given in [BPS10].

In the univariate case, there is, of course, just one choice for $R$, and (1.3) reduces to the classical three term recurrence. For the multivariate Jacobi polynomials, we propose a natural $R$, which, e.g., shares symmetries of the weight and varies continuously with it.

\section{The Jacobi polynomials on a simplex}

Here we define the Jacobi polynomials on a simplex. The orthogonal polynomials for other weight functions (or measures) on $\mathbb{R}^{d}$ can be defined similarly (see [DX01]). Applications of these polynomials include spectral methods on triangular domains [D91], Fourier series [X10], and the construction and analysis of good points for cubature and polynomial interpolation on a triangle [B83], [BS92].

Throughout, let $\xi=\left(\xi_{v}\right)_{v \in V}$ be the barycentric coordinates of the $d$-simplex $T \subset \mathbb{R}^{d}$ obtained by taking the convex hull of $d+1$ affinely independent points $V$ in $\mathbb{R}^{d}$. These are the coefficients of $x \in \mathbb{R}^{d}$ as an affine combination of the points $V$, i.e.,

$$
x=\sum_{v \in V} \xi_{v}(x) v, \quad \sum_{v \in V} \xi_{v}(x)=1,
$$

and are well suited to describing polynomials on $T$ (cf [B87]).

Cases of interest include $V=\{-1,1\} \subset \mathbb{R}^{1}$, where $T$ is the interval $[-1,1]$, and

$$
\xi_{-1}(x)=\frac{1-x}{2}, \quad \xi_{1}(x)=\frac{1+x}{2}
$$

and $V=\left\{e_{1}, e_{2}, 0\right\} \subset \mathbb{R}^{2}, e_{1}=(1,0), e_{2}=(0,1)$, where $T$ is the standard triangle

$$
T=\{(x, y): x \geq 0, y \geq 0, x+y \leq 1\},
$$


and

$$
\xi_{e_{1}}(x, y)=x, \quad \xi_{e_{2}}(x, y)=y, \quad \xi_{0}(x, y)=1-x-y .
$$

We will repeatedly use the facts that

$$
\xi_{v}(x) \geq 0, \quad \forall x \in T, \quad \xi_{v} \in \Pi_{1}\left(\mathbb{R}^{d}\right), \quad \sum_{v \in V} \xi_{v}=1 .
$$

For the parameters $\kappa=\left(\kappa_{v}\right)_{v \in V}, \kappa_{v}>-1$ we define an inner product by

$$
\langle f, g\rangle:=\int_{T} f g \xi^{\kappa}, \quad \forall f, g \in C(T) .
$$

The condition $\kappa_{v}>-1$ ensures the integrability of the Jacobi weight $\xi^{\kappa}=\prod_{v \in V} \xi_{v}^{\kappa_{v}}$. For $V=\{-1,1\} \subset \mathbb{R}$ and $\kappa_{-1}=\alpha, \kappa_{1}=\beta$, this is the usual Jacobi weight

$$
\xi^{\kappa}(x)=\left(\frac{1-x}{2}\right)^{\alpha}\left(\frac{1+x}{2}\right)^{\beta} .
$$

We will use standard multi-index notation, with $|\kappa|:=\sum_{v} \kappa_{v}$ (even if some entries are negative). The shorthand notation $1=(1)$ for the vector of 1's, etc, is used, and causes no confusion as it is easily inferred from the context. By default all multi-indices $\alpha, \beta$, etc, are in $\mathbb{Z}_{+}^{V}$, and we write the $v$-th coordinate of $\alpha$ as $\alpha_{v}$. The multivariate factorial is $\beta !:=\prod_{v} \beta_{v}$ !, and the multivariate Pochhammer symbol is $(\kappa)_{\beta}:=\prod_{v}\left(\kappa_{v}\right)_{\beta_{v}}$, with

$$
(x)_{0}:=1, \quad(x)_{n}:=x(x+1) \cdots(x+n-1), \quad x \in \mathbb{R} .
$$

Let $e_{v}$ be the multi-index which is zero in all coordinates but the $v$-th where it is 1 . By $\alpha \geq \beta$ we mean $\alpha_{v} \geq \beta_{v}, \forall v$, and similarly.

For a given $\kappa>-1$, the space $\mathcal{P}_{n}$ of (multivariate) Jacobi polynomials $\mathcal{P}_{n}$ with respect to (2.4) consists of all $f \in \Pi_{n}\left(\mathbb{R}^{d}\right)$ which satisfy

$$
\langle f, p\rangle=0, \quad \forall p \in \Pi_{n-1} .
$$

This space has dimension $\left(\begin{array}{c}n+d-1 \\ d-1\end{array}\right)$, and is spanned by the polynomials (cf [CW02], [X05])

$$
p_{\xi^{\alpha}}=p_{\xi^{\alpha}}^{\kappa}=\frac{(-1)^{|\alpha|}(\kappa+1)_{\alpha}}{(|\alpha|+|\kappa|+d)_{|\alpha|}} \sum_{\beta \leq \alpha} \frac{(|\alpha|+|\kappa|+d)_{|\beta|}(-\alpha)_{\beta}}{(\kappa+1)_{\beta}} \frac{\xi^{\beta}}{\beta !} \in \xi^{\alpha}+\Pi_{|\alpha|-1}
$$

where $|\alpha|=n$. The formula (2.5) can be expressed in terms of the Lauricella function $F_{A}$. These polynomials are the orthogonal projection of $\xi^{\alpha}$ onto $\Pi_{|\alpha|}$, and date back to Appell 1882 for the case $d=2$ and one entry of $\alpha$ fixed to be zero (cf [EMOT53] Vol 1. $§ 5.13$ and Vol. 2 \$12.4). Though not linearly independent, they do form a tight frame for $\mathcal{P}_{n}$ (cf [W06]), i.e.,

$$
f=\sum_{|\alpha|=n} \frac{(|\kappa+1|)_{2 n}}{\alpha !(\kappa+1)_{\alpha}}\left\langle f, p_{\xi^{\alpha}}\right\rangle p_{\xi^{\alpha}}, \quad \forall f \in \mathcal{P}_{n}
$$


From (2.5), we obtain

$$
p_{\xi^{\alpha}}=\sum_{\beta} c(\alpha, \beta) \xi^{\beta}, \quad c(\alpha, \beta):=\frac{(\kappa+1+\beta)_{\alpha-\beta}}{(|\alpha|+|\kappa|+d+|\beta|)_{|\alpha|-|\beta|}} \frac{(-\alpha)_{\alpha-\beta}}{(\alpha-\beta) !}
$$

where only the terms with $\beta \leq \alpha$ are nonzero. In particular, we observe that

$$
c(\alpha, \alpha-\beta)=\frac{(\kappa+1+\alpha-\beta)_{\beta}}{(2|\alpha|+|\kappa|+d-|\beta|)_{|\beta|}} \frac{(-\alpha)_{\beta}}{\beta !}, \quad 0 \leq \beta \leq \alpha .
$$

When $\xi^{\alpha}$ is the power of a barycentric coordinate $\xi_{v}$, i.e., $\alpha=n e_{v}$, the sum in $(2.5)$ becomes a ${ }_{2} F_{1}$, which can be expressed in terms of a univariate Jacobi polynomial

$$
\begin{aligned}
p_{\xi_{v}^{n}} & =\frac{(-1)^{n} n !}{(n+|\kappa|+d)_{n}} \frac{\left(\kappa_{v}+1\right)_{n}}{n !}{ }_{2} F_{1}\left(\begin{array}{c}
-n, 1+\kappa_{v}+\left(|\kappa|-\kappa_{v}+d-1\right)+n \\
\kappa_{v}+1
\end{array} ; \xi_{v}\right) \\
& =\frac{n !}{(n+|\kappa|+d)_{n}} P_{n}^{\left(|\kappa|-\kappa_{v}+d-1, \kappa_{v}\right)}\left(2 \xi_{v}-1\right) .
\end{aligned}
$$

This leads to a natural recursive three term recurrence for $p_{\xi_{v}^{n}}$.

Lemma 2.10. We have the following three term recurrence

$$
p_{\xi_{v}^{n+1}}=\left(\xi_{v}+b_{v, n}\right) p_{\xi_{v}^{n}}-c_{v, n} p_{\xi_{v}^{n-1}}, \quad n \geq 1
$$

where $b_{v, n}$ and $c_{v, n}$ are unique, and given by

$$
\begin{gathered}
b_{v, n}=-\frac{\left\langle\xi_{v} p_{\xi_{v}^{n}}, p_{\xi_{v}^{n}}\right\rangle}{\left\langle p_{\xi_{v}^{n}}, p_{\xi_{v}^{n}}\right\rangle}=\frac{\left(\kappa_{v}+n\right) n}{2 n+|\kappa|+d-1}-\frac{\left(\kappa_{v}+n+1\right)(n+1)}{2 n+|\kappa|+d+1}, \\
c_{v, n}=\frac{\left\langle p_{\xi_{v}^{n}}, p_{\xi_{v}^{n}}\right\rangle}{\left\langle p_{\xi_{v}^{n-1},}, p_{\xi_{v}^{n-1}}\right\rangle}=\frac{n\left(n+|\kappa|-\kappa_{v}+d-1\right)\left(n+\kappa_{v}\right)(n+|\kappa|+d-1)}{(2 n+|\kappa|+d-1)^{2}(2 n+|\kappa|+d-2)(2 n+|\kappa|+d)} .
\end{gathered}
$$

Proof: It follows from (2.9) and the three term recurrence relation for univariate Jacobi polynomials that $(2.11)$ holds with $b_{v, n}$ and $c_{v, n}$ given by the above formulas.

The polynomials $\left\{p_{\xi^{\alpha}}:|\alpha|=n\right\}$ given by (2.5) do not form a basis. However, the subset of those not involving the barycentric coordinate $\xi_{v_{0}}$ for a fixed point $v_{0} \in V$, i.e.,

$$
\mathcal{B}_{v_{0}, n}:=\left\{p_{\xi^{\beta}}:|\beta|=n, \beta_{v_{0}}=0\right\}
$$

do form a basis for $\mathcal{P}_{n}$. For the standard triangle (or simplex) it is usual to choose $v_{0}=0$.

Our three term recurrence formulas will be defined for all $p_{\xi^{\alpha}}$. To verify that these do give linear maps, we will need the following Lemma. 
Lemma 2.15. Fix a point $v_{0} \in V$. The polynomial $p_{\xi^{\alpha}} \in \mathcal{P}_{n}$ can be expanded in terms of the basis (2.14) as follows

$$
p_{\xi^{\alpha}}=(-1)^{\alpha_{v_{0}}} \sum_{\substack{|\beta|=\alpha_{v_{0}} \\
\beta v_{0}=0}}\left(\begin{array}{c}
\alpha_{v_{0}} \\
\beta
\end{array}\right) p_{\xi^{\beta+\alpha-\alpha_{v_{0}} e_{0}}} .
$$

In particular, for $v \neq v_{0}$, we have

$$
\begin{aligned}
(-1)^{\alpha_{v_{0}}-j} \sum_{\substack{|\beta|=\alpha_{v_{0}} \\
\beta_{v_{0}}=0}}\left(\begin{array}{c}
\alpha_{v_{0}} \\
\beta
\end{array}\right) & \left(\beta_{v}-j+1\right)_{j} p_{\xi^{\beta+\alpha-\alpha_{v_{0}} e_{v_{0}}-e_{v}}} \\
= & \left(\alpha_{v_{0}}-j+1\right)_{j} p_{\xi^{\alpha-e_{v}+j\left(e_{v}-e_{v_{0}}\right)}, \quad j \geq 0},
\end{aligned}
$$

except for when $\alpha_{v}=0$ and $j=0$.

Proof: $\quad$ Let $Q$ be the orthogonal projection onto $\mathcal{P}_{n}$. Since $Q\left(\Pi_{n-1}\right)=0$, we have

$$
p_{\xi^{\alpha}}=Q\left(\xi^{\alpha}\right)=Q\left(\left(1-\sum_{v \neq v_{0}} \xi_{v}\right)^{\alpha_{v_{0}}} \xi^{\alpha-\alpha_{v_{0}} e_{v_{0}}}\right)=Q\left(\left(-\sum_{v \neq v_{0}} \xi_{v}\right)^{\alpha_{v_{0}}} \xi^{\alpha-\alpha_{v_{0}} e_{v_{0}}}\right) .
$$

The multinomial expansion gives

$$
\left(-\sum_{v \neq v_{0}} \xi_{v}\right)^{\alpha_{v_{0}}}=(-1)^{\alpha_{v_{0}}} \sum_{\substack{|\beta|=\alpha_{v_{0}} \\
\beta_{v_{0}}=0}}\left(\begin{array}{c}
\alpha_{v_{0}} \\
\beta
\end{array}\right) \xi^{\beta}
$$

and so we conclude that

$$
p_{\xi^{\alpha}}=(-1)^{\alpha_{v_{0}}} \sum_{\substack{|\beta|=\alpha_{v_{0}} \\
\beta_{v_{0}}=0}}\left(\begin{array}{c}
\alpha_{v_{0}} \\
\beta
\end{array}\right) Q\left(\xi^{\beta+\alpha-\alpha_{v_{0}} e_{v_{0}}}\right),
$$

which is (2.16).

Now the particular case. Since

$$
\left(\begin{array}{c}
\alpha_{v_{0}} \\
\beta
\end{array}\right)\left(\beta_{v}-j+1\right)_{j}=\left(\alpha_{v_{0}}-j+1\right)_{j}\left(\begin{array}{c}
\alpha_{v_{0}}-j \\
\beta-j e_{v}
\end{array}\right)
$$

the left hand side of (2.17) becomes

$$
\left(\alpha_{v_{0}}-j+1\right)_{j}\left\{(-1)^{\alpha_{v_{0}}-j} \sum_{\substack{|\beta|=\alpha_{v_{0}} \\
\beta v_{0}=0}}\left(\begin{array}{c}
\alpha_{v_{0}}-j \\
\beta-j e_{v}
\end{array}\right) p_{\left.\xi^{\beta+\alpha-\alpha_{v_{0}} e_{v_{0}}-e_{v}}\right\}}\right\}
$$

Since either $j=0, \alpha_{v}>0$ or $j>0$, by making the substitutions

$$
\gamma=\alpha-e_{v}+j\left(e_{v}-e_{v_{0}}\right), \quad \hat{\beta}=\beta-j e_{v},
$$

the sum in the curly brackets above can be written

$$
(-1)^{\gamma_{v_{0}}} \sum_{\substack{|\hat{\beta}|=\gamma_{v_{0}} \\
\hat{\beta}_{v_{0}}=0}}\left(\begin{array}{c}
\gamma_{v_{0}} \\
\hat{\beta}
\end{array}\right) p_{\xi^{\hat{\beta}+\gamma-\gamma_{v_{0}} e_{v_{0}}}},
$$

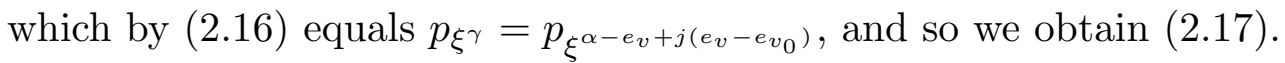




\section{Motivating examples}

Before giving the general Theorem 4.3, we illustrate the reasoning which lead to it. Here we consider the Legendre polynomials on a triangle, i.e, the constant weight $(\kappa=0)$ on a triangle $(d=2)$. We will work with the barycentric coordinates $(2.1)$.

Example 1. There are many recursive three term recurrences. We have

$$
\operatorname{span}\left(\Pi_{1} \mathcal{P}_{2}\right)=\operatorname{span}\left\{\xi_{v} p_{\xi_{w}^{2}}: v, w \in V\right\}=\mathcal{P}_{3} \oplus \mathcal{P}_{2} \oplus \mathcal{P}_{1}
$$

This follows since $\operatorname{span}\left(\Pi_{1} \mathcal{P}_{2}\right)$ is orthogonal to $\mathcal{P}_{0}$, so that $\operatorname{span}\left(\Pi_{1} \mathcal{P}_{2}\right) \subset \mathcal{P}_{3} \oplus \mathcal{P}_{2} \oplus \mathcal{P}_{1}$, and the (easily checked) fact that the nine polynomials in (3.1) are linearly independent, and hence are a basis for $\mathcal{P}_{3} \oplus \mathcal{P}_{2} \oplus \mathcal{P}_{1}$. Thus the sum $\operatorname{span}\left(\Pi_{1} \mathcal{P}_{2}\right)+\mathcal{P}_{1}$ is not direct, and there are many different three term recurrences for the cubic Legendre polynomials on a triangle (as outlined in the introduction). In particular, since $\mathcal{P}_{3} \subset \operatorname{span}\left(\Pi_{1} \mathcal{P}_{2}\right)$, there is a unique one involving only the quadratics $\mathcal{P}_{2}$. As an example, we have

$$
p_{\xi_{v}^{3}}=\frac{8}{5} \xi_{v}\left(p_{\xi_{u}^{2}}+p_{\xi_{w}^{2}}\right)+\frac{3}{5}\left(\xi_{w} p_{\xi_{w}^{2}}+\xi_{u} p_{\xi_{u}^{2}}\right)-\frac{7}{5}\left(\xi_{w} p_{\xi_{u}^{2}}+\xi_{u} p_{\xi_{w}^{2}}\right)-\frac{24}{35} p_{\xi_{v}^{2}}
$$

where $\{v, w, u\}$ are different vertices of the triangle. An even more spectacular departure from the univariate setting is that (3.1) implies $\mathcal{P}_{1}$ can be computed from $\mathcal{P}_{2}$, explicitly

$p_{\xi_{v}}=20 \xi_{v} p_{\xi_{v}^{2}}+\frac{10}{3}\left(\xi_{u}+\xi_{w}\right) p_{\xi_{v}^{2}}+\frac{70}{3}\left(\xi_{w} p_{\xi_{u}^{2}}+\xi_{u} p_{\xi_{w}^{2}}\right)-10\left(\xi_{w} p_{\xi_{w}^{2}}+\xi_{u} p_{\xi_{u}^{2}}\right)-\frac{80}{3} \xi_{v}\left(p_{\xi_{u}^{2}}+p_{\xi_{w}^{2}}\right)$.

Though the formulas of Example 1 have a certain uniqueness, it would be hard to argue that the three term recurrence (3.2) had merit over that from Lemma 2.10, i.e.,

$$
p_{\xi_{v}^{3}}=\xi_{v} p_{\xi_{v}^{2}}-\frac{17}{35} p_{\xi_{v}^{2}}-\frac{3}{50} p_{\xi_{v}}
$$

We therefore seek to extend the formula of Lemma 2.10 to all of $\mathcal{P}_{n+1}$. Below we use $B_{n}$ and $C_{n}$ for a recursive three term recurrence operator pair as in (1.2).

Example 2. The quadratics. The polynomials $\left\{p_{\xi_{v}^{2}}\right\}_{v \in V}$ are a basis for $\mathcal{P}_{2}$, on which (2.11) gives a recursive three term recurrence

$$
p_{\xi_{v}^{2}}=\left(\xi_{v}-\frac{7}{15}\right) p_{\xi_{v}}-\frac{1}{18}=: B_{1}\left(p_{\xi_{v}^{2}}\right)+C_{1}\left(p_{\xi_{v}^{2}}\right)
$$

A three term recurrence formula for $p_{\xi_{v} \xi_{w}}, v \neq w$, can be obtained by factoring out one of the barycentric coordinates

$$
p_{\xi_{v} \xi_{w}}=\left(\xi_{v}-\frac{1}{5}\right) p_{\xi_{w}}+\frac{2}{15} p_{\xi_{v}}+\frac{1}{36}, \quad p_{\xi_{v} \xi_{w}}=\left(\xi_{w}-\frac{1}{5}\right) p_{\xi_{v}}+\frac{2}{15} p_{\xi_{w}}+\frac{1}{36}
$$


Since $2 p_{\xi_{v} \xi_{w}}=p_{\xi_{u}^{2}}-p_{\xi_{v}^{2}}-p_{\xi_{w}^{2}}$, the three term recurrence given by (3.3) is

$$
\begin{aligned}
p_{\xi_{v} \xi_{w}} & =B_{1}\left(p_{\xi_{v} \xi_{w}}\right)+C_{1}\left(p_{\xi_{v} \xi_{w}}\right)=\frac{1}{2} B_{1}\left(p_{\xi_{u}^{2}}-p_{\xi_{v}^{2}}-p_{\xi_{w}^{2}}\right)+\frac{1}{2} C_{1}\left(p_{\xi_{u}^{2}}-p_{\xi_{v}^{2}}-p_{\xi_{w}^{2}}\right) \\
& =\frac{1}{2}\left(\xi_{u}-\frac{7}{15}\right) p_{\xi_{u}}-\frac{1}{2}\left(\xi_{v}-\frac{7}{15}\right) p_{\xi_{v}}-\frac{1}{2}\left(\xi_{w}-\frac{7}{15}\right) p_{\xi_{w}}+\frac{1}{2}\left(-\frac{1}{18}+\frac{1}{18}+\frac{1}{18}\right) .
\end{aligned}
$$

Using $\xi_{u}+\xi_{v}+\xi_{w}=1$ and $p_{\xi_{u}}+p_{\xi_{v}}+p_{\xi_{w}}=0$, this simplifies to

$$
p_{\xi_{v} \xi_{w}}=\frac{1}{2}\left(\xi_{v}-\frac{1}{15}\right) p_{\xi_{w}}+\frac{1}{2}\left(\xi_{w}-\frac{1}{15}\right) p_{\xi_{v}}+\frac{1}{36}
$$

which is the average of the formulas in (3.4), and has the symmetries we would expect.

Example 3. The cubics. We now consider how the recursive three term recurrence

$$
p_{\xi_{v}^{3}}=\left(\xi_{v}-\frac{17}{35}\right) p_{\xi_{v}^{2}}-\frac{3}{50} p_{\xi_{v}}=: B_{2}\left(p_{\xi_{v}^{3}}\right)+C_{2}\left(p_{\xi_{v}^{3}}\right) .
$$

can be extended. Since $3 p_{\xi_{u} \xi_{v} \xi_{w}}=p_{\xi_{u}^{3}}+p_{\xi_{v}^{3}}+p_{\xi_{w}^{3}}$, we obtain from (3.6) that

$$
p_{\xi_{u} \xi_{v} \xi_{w}}=\left(B_{2}+C_{2}\right)\left(p_{\xi_{u} \xi_{v} \xi_{w}}\right)=\frac{1}{3}\left(\xi_{u}-\frac{17}{35}\right) p_{\xi_{u}^{2}}+\frac{1}{3}\left(\xi_{v}-\frac{17}{35}\right) p_{\xi_{v}^{2}}+\frac{1}{3}\left(\xi_{w}-\frac{17}{35}\right) p_{\xi_{w}^{2}}+0 .
$$

Thus we need only decide on the best recurrence for $p_{\xi_{v}^{2}} \xi_{w}$. The analogue of (3.4) is

$$
\begin{aligned}
& p_{\xi_{v}^{2} \xi_{w}}=\left(\xi_{v}-\frac{13}{35}\right) p_{\xi_{v} \xi_{w}}+\frac{2}{35} p_{\xi_{v}^{2}}+\frac{1}{60} p_{\xi_{v}}-\frac{2}{75} p_{\xi_{w}}=: f_{1}^{v, w}(\xi), \\
& p_{\xi_{v}^{2} \xi_{w}}=\left(\xi_{w}-\frac{1}{7}\right) p_{\xi_{v}^{2}}+\frac{8}{35} p_{\xi_{v} \xi_{w}}+\frac{2}{75} p_{\xi_{v}}-\frac{1}{150} p_{\xi_{w}}=: f_{2}^{v, w}(\xi) .
\end{aligned}
$$

The four dimensional space $\mathcal{P}_{3}$ can be decomposed

$$
\mathcal{P}_{3}=\operatorname{span}\left\{p_{\xi_{v}^{3}}: v \in V\right\} \oplus \operatorname{span}\{q\},
$$

where, with $v, w, u$ some fixed ordering of the points of $V$,

$$
q=q^{v, w, u}:=p_{\xi_{v}}\left(\xi_{w}^{2}-\xi_{u}^{2}\right)+\xi_{w}\left(\xi_{u}^{2}-\xi_{v}^{2}\right)+\xi_{u}\left(\xi_{v}^{2}-\xi_{w}^{2}\right) .
$$

The polynomial $q$ above is mapped to itself by rotations of the triangle, and to its negative by the reflections. Hence for a recurrence to be invariant under the symmetries of the triangle, one must choose the space spanned by the linear polynomial $C_{2}(q) \in \mathcal{P}_{1}=\Pi_{1} \ominus \Pi_{0}$ to be invariant under these symmetries, i.e., $C_{2}(q)=0$, giving $q=B_{2}(q)$. By (3.1) this is possible, with the formula given by

$$
B_{2}(q)=q=\xi_{v}\left(p_{\xi_{w}^{2}}-p_{\xi_{u}^{2}}\right)+\xi_{w}\left(p_{\xi_{u}^{2}}-p_{\xi_{v}^{2}}\right)+\xi_{u}\left(p_{\xi_{v}^{2}}-p_{\xi_{w}^{2}}\right) \in \operatorname{span}\left(\Pi_{1} \mathcal{P}_{2}\right) .
$$

Since

$$
p_{\xi_{v}^{2} \xi_{w}}=-\frac{1}{6} p_{\xi_{v}\left(\xi_{w}^{2}-\xi_{u}^{2}\right)+\xi_{w}\left(\xi_{u}^{2}-\xi_{v}^{2}\right)+\xi_{u}\left(\xi_{v}^{2}-\xi_{w}^{2}\right)}-\frac{1}{2} p_{\xi_{v}^{3}}+\frac{1}{6} p_{\xi_{w}^{3}}-\frac{1}{6} p_{\xi_{u}^{3}}
$$


we can use (3.6) and (3.9) to obtain the corresponding recurrence for $p_{\xi_{v}^{2} \xi_{w}}$

$$
C_{2}\left(p_{\xi_{v}^{2} \xi_{w}}\right)=-\frac{1}{6} 0-\frac{1}{2}\left(-\frac{3}{50} p_{\xi_{v}}\right)+\frac{1}{6}\left(-\frac{3}{50} p_{\xi_{w}}\right)-\frac{1}{6}\left(-\frac{3}{50} p_{\xi_{u}}\right)=\frac{1}{50}\left(p_{\xi_{v}}-p_{\xi_{w}}\right),
$$

since $p_{\xi_{v}}+p_{\xi_{w}}+p_{\xi_{u}}=0$, and after considerable (but routine) simplification

$$
B_{2}\left(p_{\xi_{v}^{2} \xi_{w}}\right)=\frac{2}{3}\left(\xi_{v}-\frac{9}{35}\right) p_{\xi_{v} \xi_{w}}+\frac{1}{3}\left(\xi_{w}-\frac{1}{35}\right) p_{\xi_{v}^{2}} .
$$

Thus the best possible recurrence for $p_{\xi_{v}^{2} \xi_{w}}$ is

$$
\begin{aligned}
p_{\xi_{v}^{2} \xi_{w}} & =\frac{2}{3}\left(\xi_{v}-\frac{9}{35}\right) p_{\xi_{v} \xi_{w}}+\frac{1}{3}\left(\xi_{w}-\frac{1}{35}\right) p_{\xi_{v}^{2}}+\frac{1}{50}\left(p_{\xi_{v}}-p_{\xi_{w}}\right) \\
& =\frac{2}{3} f_{1}^{v, w}(\xi)+\frac{1}{3} f_{2}^{v, w}(\xi),
\end{aligned}
$$

which we observe is (remarkably) a convex combination of those in (3.8).

It turns out that extensions of Lemma 2.10, similar to that of (3.10), hold for the Jacobi polynomials of any degree for any weight in any number of dimensions (Theorem 4.3). The general form of the recurrences (3.3) and (3.6) is given in Proposition 5.1.

\section{The recursive three term recurrence}

We now extend the best possible recurrence formula (3.10) for the cubic Legendre polynomials on a triangle. The key features of our recurrence are:

- The formulas are simple, recursive, and reduce to those of Lemma 2.10.

- They are defined on the linearly dependent set $\left\{p_{\xi^{\alpha}}\right\}$, and give linear maps.

- They have the same symmetries as the spaces they are defined on.

- They depend continuously on the parameter $\kappa>-1$.

Recall that an affine map $\sigma$ of the simplex $T$ onto itself induces an action on functions

$$
\sigma f:=f\left(\sigma^{-1}\right), \quad f \in C(T),
$$

and is uniquely determined by its action on the vertices $V$ (a permutation of $V$ ), or, equivalently, its (induced) action on the barycentric coordinates. We observe

$$
\sigma \xi_{v}=\xi_{\sigma v}, \quad \forall v \in V, \quad \Longrightarrow \quad \sigma \xi^{\alpha}=\xi^{\sigma \alpha}, \quad \sigma \alpha:=\left(\alpha_{\sigma^{-1} v}\right)_{v \in V} .
$$

The symmetry group of a Jacobi weight $\xi^{\kappa}$ consists of those affine maps $\sigma$ which map $T$ onto itself and leave $\xi^{\kappa}$ invariant, i.e.,

$$
G=\operatorname{Sym}\left(\xi^{\kappa}\right):=\left\{\sigma: \sigma \xi^{\kappa}=\xi^{\kappa}\right\}=\left\{\sigma: \kappa_{\sigma v}=\kappa_{v}, \forall v \in V\right\} .
$$

Clearly, this is a group, and $\mathcal{P}_{n}$ is invariant under its action, i.e., for all $\sigma \in \operatorname{Sym}\left(\xi^{\kappa}\right)$

$$
\sigma f \in \mathcal{P}_{n}, \quad \forall f \in \mathcal{P}_{n},
$$

since (by a change of variables) $\langle\sigma f, \sigma g\rangle=\langle f, g\rangle, \forall f, g$.

We denote the support of a multi-index $\beta$ by $\operatorname{supp} \beta:=\left\{v \in V: \beta_{v}>0\right\}$. 
Theorem 4.3 (Recursive three term recurrence). Let $n \geq 0, \lambda:=2|\alpha|+|\kappa|+d-3$. Then the Jacobi polynomials $p_{\xi^{\alpha}} \in \mathcal{P}_{n+1}$ satisfy the (recursive) three term recurrence

$$
p_{\xi^{\alpha}}=\sum_{v \in V} \frac{\alpha_{v}}{|\alpha|}\left(\xi_{v}+b_{v}^{\alpha}\right) p_{\xi^{\alpha} / \xi_{v}}-\sum_{\substack{|\beta|=2 \\ \beta \leq \alpha}} c_{\beta}^{\alpha} p_{\xi^{\alpha-\beta}}, \quad \alpha \in \mathbb{Z}_{V}^{+}, \quad|\alpha|=n+1,
$$

where $b_{v}^{\alpha}$ and $c_{\beta}^{\alpha}$ are the continuous functions of $\kappa$ given by

$$
b_{v}^{\alpha}:=\frac{\left(\kappa_{v}+\alpha_{v}\right)(|\alpha|-1)-\alpha_{v}+1}{2|\alpha|+|\kappa|+d-3}-\frac{|\alpha|\left(\kappa_{v}+\alpha_{v}\right)}{2|\alpha|+|\kappa|+d-1}, \quad \alpha_{v} \neq 0
$$

except for $\lambda=0$, i.e., $|\alpha|=1,|\kappa|=-d+1$, and

$$
c_{\beta}^{\alpha}:=\frac{(\kappa+1+\alpha-\beta)_{\beta}}{|\alpha| \lambda^{2}(\lambda-1)} \frac{(-\alpha)_{\beta}}{\beta !}\left\{\frac{2|\alpha|}{\lambda+1}-2+\sum_{v \in \operatorname{supp} \beta} \beta_{v} \frac{\lambda\left(\beta_{v}-1\right)+1-\alpha_{v}}{\kappa_{v}+\alpha_{v}}\right\},
$$

except for $\lambda=1$, i.e., $|\alpha|=2,|\kappa|=-d$. Moreover, there are (unique) linear maps $B_{n}=B_{n}^{\kappa}: \mathcal{P}_{n+1} \rightarrow \operatorname{span}\left\{\Pi_{1} \mathcal{P}_{n}\right\}$ and $C_{n}=C_{n}^{\kappa}: \mathcal{P}_{n+1} \rightarrow \mathcal{P}_{n-1}$ with

$$
B_{n}\left(p_{\xi^{\alpha}}\right)=\sum_{v \in V} \frac{\alpha_{v}}{|\alpha|}\left(\xi_{v}+b_{v}^{\alpha}\right) p_{\xi^{\alpha} / \xi_{v}}, \quad C_{n}\left(p_{\xi^{\alpha}}\right)=\sum_{\substack{|\beta|=2 \\ \beta \leq \alpha}} c_{\beta}^{\alpha} p_{\xi^{\alpha-\beta}}, \quad \forall p_{\xi^{\alpha}} \in \mathcal{P}_{n+1}
$$

and these commute with the action of the symmetries of the weight.

Proof: The formal power series in $\xi$ obtained by substituting (2.7) into

$$
p_{\xi^{\alpha}}-\sum_{v \in V} \frac{\alpha_{v}}{|\alpha|}\left(\xi_{v}+b_{v}^{\alpha}\right) p_{\xi^{\alpha} / \xi_{v}}
$$

is a polynomial of degree $n$ involving only the powers $\xi^{\beta}, \beta<\alpha$. The coefficient of $\xi^{\alpha-e_{v}}$ is

$$
c\left(\alpha, \alpha-e_{v}\right)-\sum_{w \in \operatorname{supp}\left(\alpha-e_{v}\right)} \frac{\alpha_{w}}{|\alpha|} c\left(\alpha-e_{w}, \alpha-e_{w}-e_{v}\right)-\frac{\alpha_{v}}{|\alpha|} b_{v}^{\alpha} .
$$

This can be made zero by choosing $b_{v}^{\alpha}\left(\right.$ for $\left.\alpha_{v} \neq 0\right)$ to be

$$
b_{v}^{\alpha}:=\frac{|\alpha|}{\alpha_{v}} c\left(\alpha, \alpha-e_{v}\right)-\frac{|\alpha|}{\alpha_{v}} \sum_{w \in \operatorname{supp}\left(\alpha-e_{v}\right)} \frac{\alpha_{w}}{|\alpha|} c\left(\alpha-e_{w}, \alpha-e_{w}-e_{v}\right) .
$$

Using (2.8), and taking care when the sum over $w \in \operatorname{supp}\left(\alpha-e_{v}\right)$ is empty, (4.9) becomes

$$
\begin{aligned}
b_{v}^{\alpha} & =-\frac{|\alpha|}{\alpha_{v}} \frac{\left(\kappa_{v}+\alpha_{v}\right) \alpha_{v}}{2|\alpha|+|\kappa|+d-1}+\sum_{w \neq v} \frac{\alpha_{w}}{\alpha_{v}} \frac{\left(\kappa_{v}+\alpha_{v}\right) \alpha_{v}}{2|\alpha|+|\kappa|+d-3}+\frac{\alpha_{v}}{\alpha_{v}} \frac{\left(\kappa_{v}+\alpha_{v}-1\right)\left(\alpha_{v}-1\right)}{2|\alpha|+|\kappa|+d-3} \\
& =-\frac{|\alpha|\left(\kappa_{v}+\alpha_{v}\right)}{2|\alpha|+|\kappa|+d-1}+\frac{\left(|\alpha|-\alpha_{v}\right)\left(\kappa_{v}+\alpha_{v}\right)}{2|\alpha|+|\kappa|+d-3}+\frac{\left(\kappa_{v}+\alpha_{v}-1\right)\left(\alpha_{v}-1\right)}{2|\alpha|+|\kappa|+d-3}
\end{aligned}
$$


except when $\lambda=0$, which gives (4.5).

With the choice (4.5) for $b_{v}^{\alpha}$, the formal power series obtained from (4.8) becomes a polynomial of degree $n-1$, with the coefficient of $\xi^{\alpha-\beta}, \beta \leq \alpha,|\beta|=2$, given by

$$
-c_{\beta}^{\alpha}:=c(\alpha, \alpha-\beta)-\sum_{\substack{v \in V \\ \alpha_{v}-\beta_{v} \geq 1}} \frac{\alpha_{v}}{|\alpha|} c\left(\alpha-e_{v}, \alpha-e_{v}-\beta\right)-\sum_{\substack{v \in V \\ \beta_{v} \geq 1}} \frac{\alpha_{v}}{|\alpha|} c\left(\alpha-e_{v}, \alpha-\beta\right) b_{v}^{\alpha},
$$

which, by Lemma 6.1, simplifies to (4.6). Here $|\alpha| \geq 2$ implies $\lambda>0$. With this definition for $c_{\beta}^{\alpha}$,

$$
p_{\xi^{\alpha}}-\sum_{v \in V} \frac{\alpha_{v}}{|\alpha|}\left(\xi_{v}+b_{v}^{\alpha}\right) p_{\xi^{\alpha} / \xi_{v}}+\sum_{\substack{|\beta|=2 \\ \beta \leq \alpha}} c_{\beta}^{\alpha} p_{\xi^{\alpha-\beta}}
$$

is a polynomial of degree $n-2$, which is orthogonal to $\Pi_{n-2}$, and hence is equal to zero. This establishes (4.4), except in the exceptional cases $\lambda=0$ and $\lambda=1$. Outside of these cases, $b_{v}^{\alpha}$ and $c_{\beta}^{\alpha}$ are rational functions of $\kappa$, and hence are continuous.

When $b_{v}^{\alpha}$ and $c_{\beta}^{\alpha}$ are not defined by (4.5) and (4.6), we extend (4.4) by taking limits in $\kappa$, as follows. Observe $\lambda=2|\alpha|+|\kappa|+d-3 \rightarrow \lambda^{*}=2|\alpha|+\left|\kappa^{*}\right|+d-3$ as $\kappa \rightarrow \kappa^{*}$. Thus, for $|\alpha|=1, \lambda \neq 0$, we have

$$
b_{v}^{\alpha}=\frac{0}{\lambda}-\frac{\kappa_{v}+1}{\lambda+2} \rightarrow-\frac{\kappa_{v}^{*}+1}{2}, \quad \text { as } \kappa \rightarrow \kappa^{*},
$$

and for $|\alpha|=2(\beta=\alpha), \lambda \neq 1$, we have

$$
\begin{aligned}
c_{\beta}^{\alpha} & =\frac{(\kappa+1)_{\alpha}}{2 \lambda^{2}}\left\{\frac{-2}{\lambda+1}+\sum_{v \in \operatorname{supp} \alpha} \frac{\alpha_{v}\left(\alpha_{v}-1\right)}{\kappa_{v}+\alpha_{v}}\right\} \\
& \rightarrow \frac{\left(\kappa^{*}+1\right)_{\alpha}}{2}\left\{-2+\sum_{v \in \operatorname{supp} \alpha} \frac{\alpha_{v}\left(\alpha_{v}-1\right)}{\kappa_{v}^{*}+\alpha_{v}}\right\}, \quad \text { as } \kappa \rightarrow \kappa^{*}
\end{aligned}
$$

By defining $b_{v}^{\alpha}$ and $c_{\beta}^{\alpha}$ to take these limiting values in the exceptional cases, (4.4) can be extended, with the $b_{v}^{\alpha}$ and $c_{\beta}^{\alpha}$ (so defined) being continuous functions of $\kappa$.

We now show that there are linear maps $B_{n}$ and $C_{n}$ on $\mathcal{P}_{n+1}$ satisfying (4.7). Since (4.4) implies the sum of such maps would be the identity, it suffices to consider only $B_{n}$. Let $B_{n}\left(p_{\xi^{\alpha}}\right)$ be defined by $(4.7)$, and $L: \mathcal{P}_{n+1} \rightarrow \operatorname{span}\left(\Pi_{1} \mathcal{P}_{n}\right)$ be the linear map defined on the basis $(2.14)$ by $L\left(p_{\xi^{\alpha}}\right):=B_{n}\left(p_{\xi^{\alpha}}\right), \alpha\left(v_{0}\right)=0$. In view of the basis expansion (2.16),

$$
L\left(p_{\xi^{\alpha}}\right)=(-1)^{\alpha_{v_{0}}} \sum_{\substack{|\beta|=\alpha_{v_{0}} \\
\beta_{v_{0}}=0}}\left(\begin{array}{c}
\alpha_{v_{0}} \\
\beta
\end{array}\right) B_{n}\left(p_{\xi^{\beta+\alpha-\alpha_{v_{0}} e_{v_{0}}}}\right)
$$

and we need only show that $L\left(p_{\xi^{\alpha}}\right)=B_{n}\left(p_{\xi^{\alpha}}\right)$ (for $\left.\alpha_{v_{0}} \neq 0\right)$.

Fix $\beta$ with $|\beta|=\alpha_{v_{0}}, \beta_{v_{0}}=0$ and $v \neq v_{0}$. Since

$$
b_{v}^{\beta+\alpha-\alpha_{v_{0}} e_{v_{0}}}=b_{v}^{\alpha}-r \beta_{v}, \quad r=r_{|\alpha|,|\kappa|, d}:=\frac{2(|\alpha|+|\kappa|+d-1)}{\lambda(\lambda+2)},
$$


we obtain

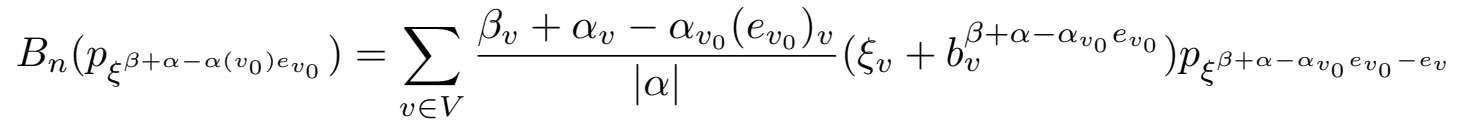

$$
\begin{aligned}
& =\sum_{v \neq v_{0}} \frac{\beta_{v}+\alpha_{v}}{|\alpha|}\left(\xi_{v}+b_{v}^{\alpha}-r \beta_{v}\right) p_{\xi^{\beta+\alpha-\alpha_{v_{0}} e_{v_{0}}-e_{v}}} \\
& =\sum_{v \neq v_{0}}\left\{\frac{\alpha_{v}}{|\alpha|}\left(\xi_{v}+b_{v}^{\alpha}\right)+\frac{\beta_{v}}{|\alpha|}\left(\xi_{v}+b_{v}^{\alpha}-r-r \alpha_{v}\right)+\frac{\beta_{v}\left(\beta_{v}-1\right)}{|\alpha|}(-r)\right\} p_{\xi^{\beta+\alpha-\alpha_{v_{0}} e_{v_{0}}-e_{v}}} .
\end{aligned}
$$

By substituting (4.12) into (4.11), interchanging the order of summation, and then applying (2.17) of Lemma 2.15 for the cases $j=0,1,2$, respectively, we obtain

$$
\begin{aligned}
L\left(p_{\xi^{\alpha}}\right)=\sum_{v \neq v_{0}} & \frac{\alpha_{v}}{|\alpha|}\left(\xi_{v}+b_{v}^{\alpha}\right) p_{\xi^{\alpha-e_{v}}}-\sum_{v \neq v_{0}} \frac{\alpha_{v_{0}}}{|\alpha|}\left(\xi_{v}+b_{v}^{\alpha}-r-r \alpha_{v}\right) p_{\xi^{\alpha-e_{v}}} \\
& -\sum_{v \neq v_{0}} \frac{r}{|\alpha|} \alpha_{v_{0}}\left(\alpha_{v_{0}}-1\right) p_{\xi^{\alpha+e_{v}-2 e_{v_{0}}}} .
\end{aligned}
$$

Since

$$
\sum_{v \neq v_{0}} \xi_{v}=1-\xi_{v_{0}}, \quad \sum_{v \neq v_{0}} b_{v}^{\alpha}=-1-b_{v_{0}}^{\alpha}+r(|\alpha|+d-1), \quad \sum_{v \neq v_{0}} p_{\xi^{\alpha+e_{v}-2 e_{v_{0}}}}=-p_{\xi^{\alpha-e_{v_{0}}}}
$$

the sum of the last two terms of (4.13) is

$$
-\frac{\alpha_{v_{0}}}{|\alpha|}\left(1-\xi_{v_{0}}-1-b_{v_{0}}^{\alpha}+r(|\alpha|+d-1)-r d-r\left(|\alpha|-\alpha_{v_{0}}\right)-r\left(\alpha_{v_{0}}-1\right)\right) p_{\xi^{\alpha-e_{v}}}
$$

and so (4.13) becomes

$$
L\left(p_{\xi^{\alpha}}\right)=\sum_{v \neq v_{0}} \frac{\alpha_{v}}{|\alpha|}\left(\xi_{v}+b_{v}^{\alpha}\right) p_{\xi^{\alpha-e_{v}}}+\frac{\alpha_{v_{0}}}{|\alpha|}\left(\xi_{v_{0}}+b_{v_{0}}^{\alpha}\right) p_{\xi^{\alpha-e_{v_{0}}}}=B_{n}\left(p_{\xi^{\alpha}}\right) .
$$

Thus the existence of a (unique) linear map $B_{n}$ satisfying (4.7) is proved.

Finally, the symmetry condition. Since $B_{n}+C_{n}$ is the identity, its suffices to show it for $B_{n}$. Since $(\sigma \alpha)_{\sigma v}=\alpha_{v},(2.5)$ gives $\sigma p_{\xi^{\alpha}}^{\kappa}=p_{\xi \sigma \alpha}^{\sigma \kappa}$. Hence for $\sigma \in \operatorname{Sym}\left(\xi^{\kappa}\right)$, we have $\sigma p_{\xi^{\alpha}}=p_{\xi^{\sigma \alpha}}$, and thereby obtain

$$
\sigma\left(B_{n} p_{\xi^{\alpha}}\right)=\sum_{v \in V} \frac{\alpha_{v}}{|\alpha|}\left(\sigma \xi_{v}+b_{v}^{\alpha}\right) \sigma p_{\xi^{\alpha} / \xi_{v}}=\sum_{v \in V} \frac{(\sigma \alpha)_{\sigma v}}{|\alpha|}\left(\xi_{\sigma v}+b_{\sigma v}^{\sigma \alpha}\right) p_{\xi^{\sigma \alpha} / \xi_{\sigma v}}=B_{n}\left(p_{\xi^{\sigma \alpha}}\right)
$$

i.e., $\sigma B_{n}=B_{n} \sigma, \forall \sigma \in \operatorname{Sym}\left(\xi^{\kappa}\right)$, as asserted. 
We note that when $b_{v}^{\alpha}$ and $c_{\beta}^{\alpha}$ are not defined by (4.5) and (4.6), they are given by

$$
b_{v}^{\alpha}=-\frac{\kappa_{v}+1}{2} \quad(\lambda=0), \quad c_{\beta}^{\alpha}=\frac{(\kappa+1) \alpha}{2}\left\{-2+\sum_{v \in \operatorname{supp} \alpha} \frac{\alpha_{v}\left(\alpha_{v}-1\right)}{\kappa_{v}+\alpha_{v}}\right\} \quad(\lambda=1) .
$$

The proof above shows (more generally) that for any permutation $\sigma$ of the vertices

$$
\sigma B_{n}^{\kappa}(f)=B_{n}^{\sigma \kappa}(\sigma f), \quad \sigma C_{n}^{\kappa}(f)=C_{n}^{\sigma \kappa}(\sigma f) .
$$

Theorem 4.3 reduces to Lemma 2.10 when $\alpha=(n+1) e_{v}$. For the linears $\mathcal{P}_{1},(4.4)$ gives

$$
p_{\xi_{v}}=\xi_{v}-\frac{\kappa_{v}+1}{|\kappa|+d+1}
$$

for the quadratics $\mathcal{P}_{2}$ it gives (3.3), (3.5), and for the cubics $\mathcal{P}_{3}$ it gives (3.6), (3.10) and (3.7) in the form

$$
p_{\xi_{v} \xi_{w} \xi_{u}}=\frac{1}{3}\left(\xi_{v}-\frac{1}{35}\right) p_{\xi_{w} \xi_{u}}+\frac{1}{3}\left(\xi_{w}-\frac{1}{35}\right) p_{\xi_{v} \xi_{u}}+\frac{1}{3}\left(\xi_{u}-\frac{1}{35}\right) p_{\xi_{v} \xi_{w}} .
$$

A basis for $\mathcal{P}_{n}$ can be obtained by projecting the monomials

$$
x \mapsto x^{\alpha}=x_{1}^{\alpha_{1}} x_{2}^{\alpha_{2}} \cdots x_{d}^{\alpha_{d}}, \quad|\alpha|=n
$$

onto $\mathcal{P}_{n}$. The resulting polynomials $P_{\alpha}(x)$, which have leading term $x^{\alpha}$, are the monic or monomial orthogonal polynomials (see [S99], [X05]). For the standard triangle we have the following.

Corollary 4.16 (Standard triangle). For the weight

$$
w(x, y)=x^{\alpha} y^{\beta}(1-x-y)^{\gamma}, \quad \alpha, \beta, \gamma>-1
$$

on the standard triangle (2.2) the monomial orthogonal polynomials $P_{j, k}(x, y)=x^{j} y^{k}+\cdots$, $j+k>0$ are given by the recursive three term recurrence

$$
\begin{aligned}
P_{j, k}(x, y)=\frac{j}{j+k} & \left(x+b_{1}^{j, k}\right) P_{j-1, k}(x, y)+\frac{k}{j+k}\left(y+b_{2}^{j, k}\right) P_{j, k-1}(x, y) \\
& -c_{2,0}^{j, k} P_{j-2, k}(x, y)-c_{1,1}^{j, k} P_{j-1, k-1}(x, y)-c_{0,2}^{j, k} P_{j, k-2}(x, y),
\end{aligned}
$$

where, $P_{0,0}(x, y):=1$, and with $\lambda:=2(j+k)+\alpha+\beta+\gamma-1$,

$$
\begin{aligned}
b_{1}^{j, k} & :=\frac{(\alpha+j)(j+k-1)-j+1}{\lambda}-\frac{(j+k)(\alpha+j)}{\lambda+2}, \\
b_{2}^{j, k} & :=\frac{(\beta+k)(j+k-1)-k+1}{\lambda}-\frac{(j+k)(\beta+k)}{\lambda+2}, \\
c_{2,0}^{j, k} & :=\frac{j(j-1)(\alpha+j-1)(\alpha+j)}{(j+k) \lambda^{2}(\lambda-1)}\left\{\frac{j+k}{\lambda+1}-1+\frac{\lambda+1-j}{\alpha+j}\right\}, \\
c_{0,2}^{j, k} & :=\frac{k(k-1)(\beta+k-1)(\beta+k)}{(j+k) \lambda^{2}(\lambda-1)}\left\{\frac{j+k}{\lambda+1}-1+\frac{\lambda+1-k}{\beta+k}\right\}, \\
c_{1,1}^{j, k} & :=\frac{j k}{j+k} \frac{(\alpha+j)(\beta+k)}{\lambda^{2}(\lambda-1)}\left\{\frac{2(j+k)}{\lambda+1}-2+\left(\frac{1-k}{\beta+k}+\frac{1-j}{\alpha+j}\right)\right\} .
\end{aligned}
$$

Proof: In view of (2.3), the monomial orthogonal polynomials are given by

$$
P_{j, k}=P_{j, k}^{(\alpha, \beta, \gamma)}:=p_{\xi^{(j, k, 0)}}, \quad \xi(x, y)=(x, y, 1-x-y), \quad \kappa=(\alpha, \beta, \gamma),
$$

to which we apply Theorem 4.3 for the multi-indices $(j, k, 0)$. 
We conclude this section with some technical remarks about Theorem 4.3. The special case $\lambda=0$ in (4.5) is already apparent in the recurrence for univariate Jacobi polynomials

$$
\begin{aligned}
P_{n+1}^{(\alpha, \beta)}(x)= & \frac{(2 n+\alpha+\beta+1)(2 n+\alpha+\beta+2)}{2(n+1)(n+\alpha+\beta+1)} x P_{n}^{(\alpha, \beta)}(x) \\
& +\frac{(2 n+\alpha+\beta+1)\left(\alpha^{2}-\beta^{2}\right)}{2(n+1)(n+\alpha+\beta+1)(2 n+\alpha+\beta)} P_{n}^{(\alpha, \beta)}(x) \\
& -\frac{(\alpha+n)(\beta+n)(2 n+\alpha+\beta+2)}{(n+1)(n+\alpha+\beta+1)(2 n+\alpha+\beta)} P_{n-1}^{(\alpha, \beta)}(x),
\end{aligned}
$$

where the denominator $\lambda=2 n+\alpha+\beta$ is zero for $n=0, \alpha+\beta=0$. This is used by [IM91] as the starting point to define the exceptional Jacobi polynomials via the recurrence (4.20). In a similar fashion, one might define multivariate exceptional Jacobi polynomials.

It appears that $c_{\beta}^{\alpha} \neq 0$ for all $\beta \leq \alpha,|\beta|=2$, specifically

$$
c_{2 e_{v}}^{\alpha}>0, \quad c_{e_{v}+e_{w}}^{\alpha}<0, \quad v \neq w,
$$

which would imply that $C_{n}$ is onto $\mathcal{P}_{n-1}$. This could be considered a multivariate analogue of $C_{n} \neq 0$. By way of comparison, $C_{2}=0$ in the recurrence of (3.7).

We emphasize that our three term recurrence is a pair of linear maps. Hence it gives explicit three term recurrence formulas for any other system (by substitution) - provided that $\left\langle f, p_{\xi^{\alpha}}\right\rangle$ can be computed for each polynomial $f$ in the system. For example, if $\left(Q_{j}^{[n]}\right)$ is an orthonormal basis for $\mathcal{P}_{n}$, then substituting our recurrence into (2.6) and expanding in the orthonormal basis gives

$$
Q_{\ell}^{[n+1]}=\sum_{j} A_{j}^{[n]} Q_{j}^{[n]}+\sum_{k} B_{k}^{[n]} Q_{k}^{[n-1]}
$$

where

$$
\begin{aligned}
& A_{j}^{[n]}:=\sum_{|\alpha|=n+1} \frac{(|\kappa+1|)_{2 n+2}}{\alpha !(\kappa+1)_{\alpha}}\left\langle Q_{\ell}^{[n+1]}, p_{\xi^{\alpha}}\right\rangle \sum_{v \in V} \frac{\alpha_{v}}{|\alpha|}\left(\xi_{v}+b_{v}^{\alpha}\right)\left\langle p_{\xi^{\alpha} / \xi_{v}}, Q_{j}^{[n]}\right\rangle, \\
& B_{k}^{[n]}:=-\sum_{|\alpha|=n+1} \frac{(|\kappa+1|)_{2 n+2}}{\alpha !(\kappa+1)_{\alpha}}\left\langle Q_{\ell}^{[n+1]}, p_{\xi^{\alpha}}\right\rangle \sum_{\substack{|\beta|=2 \\
\beta \leq \alpha}} c_{\beta}^{\alpha}\left\langle p_{\xi^{\alpha-\beta}}, Q_{k}^{[n-1]}\right\rangle .
\end{aligned}
$$

The formula (4.21) for an orthonormal basis (cf [P57]), and simplifications of it, gives a benchmark with which to compare any other three term recurrence for an orthogonal basis that might be constructed in the future.

\section{Nonrecursive three term recurrences}

We now give an explicit formula for the inclusion

$$
\operatorname{span}\left(\Pi_{1} \mathcal{P}_{n}\right) \subset \mathcal{P}_{n+1} \oplus \mathcal{P}_{n} \oplus \mathcal{P}_{n-1}
$$

This extends three term recurrence of [BS92] for the monomial Jacobi polynomials on the standard triangle (cf Corollary 4.16). 
Proposition 5.1 (Three term recurrence). Let $n \geq 0$, and $\lambda:=2|\alpha|+|\kappa|+d-3$. Then the Jacobi polynomials $p_{\xi^{\alpha}} \in \mathcal{P}_{n+1}$ satisfy the (nonrecursive) three term recurrence

$$
\xi_{v} p_{\xi^{\alpha} / \xi_{v}}=p_{\xi^{\alpha}}-\sum_{w \in \operatorname{supp} \alpha} b_{w}^{\alpha, v} p_{\xi^{\alpha-e_{w}}}+\sum_{\substack{|\beta|=2 \\ \beta \leq \alpha}} c_{\beta}^{\alpha, v} p_{\xi^{\alpha-\beta}}, \quad \alpha_{v} \neq 0
$$

where $b_{w}^{\alpha, v}$ and $c_{\beta}^{\alpha, v}$ are the continuous functions of $\kappa$ given by

$$
b_{w}^{\alpha, v}:=\frac{2 \alpha_{w}\left(\kappa_{w}+\alpha_{w}\right)}{\lambda(\lambda+2)}, \quad w \neq v, \quad b_{v}^{\alpha, v}:=\frac{\left(\alpha_{v}-1\right)\left(\kappa_{v}+\alpha_{v}-1\right)}{\lambda}-\frac{\alpha_{v}\left(\kappa_{v}+\alpha_{v}\right)}{\lambda+2} .
$$

except for $\lambda=0$, i.e., $|\alpha|=1,|\kappa|=-d+1$, and

$$
c_{\beta}^{\alpha, v}:=\frac{(\kappa+1+\alpha-\beta)_{\beta}}{\lambda^{2}(\lambda-1)} \frac{(-\alpha)_{\beta}}{\beta !}\left\{\frac{2}{\lambda+1}-\frac{\beta_{v}}{\alpha_{v}}+\frac{\beta_{v}}{\alpha_{v}} \frac{\lambda\left(\beta_{v}-1\right)+1-\alpha_{v}}{\kappa_{v}+\alpha_{v}}\right\},
$$

except for $\lambda=1$, i.e., $|\alpha|=2,|\kappa|=-d$.

Proof: In a similar fashion to Theorem 4.3, choose

$$
b_{w}^{\alpha, v}=c\left(\alpha, \alpha-e_{w}\right)-c\left(\alpha-e_{v}, \alpha-e_{v}-e_{w}\right)=\frac{\left(\alpha_{w}-\delta_{v w}\right)\left(\kappa_{w}+\alpha_{w}-\delta_{v w}\right)}{\lambda}-\frac{\alpha_{w}\left(\kappa_{w}+\alpha_{w}\right)}{\lambda+2},
$$

where $\delta_{v w}=\left(e_{v}\right)_{w}$ is the Dirac delta function, so that

$$
\xi_{v} p_{\xi^{\alpha} / \xi_{v}}-\left(p_{\xi^{\alpha}}-\sum_{w \in \operatorname{supp} \alpha} b_{w}^{\alpha, v} p_{\xi^{\alpha-e_{w}}}\right)
$$

is a polynomial of degree $|\alpha|-2$, and (5.3) holds. Let $c_{\beta}^{\alpha, v}$ be the $\xi^{\alpha-\beta}(\beta \leq \alpha,|\beta|=2)$ coefficient of formal power series (5.6) in $\xi$, i.e.,

$$
c_{\beta}^{\alpha, v}=c\left(\alpha-e_{v}, \alpha-e_{v}-\beta\right)-c(\alpha, \alpha-\beta)+\sum_{w \in \operatorname{supp} \beta} b_{w}^{\alpha, v} c\left(\alpha-e_{w}, \alpha-\beta\right),
$$

which, by Lemma 6.1, simplifies to (5.4). With this definition for $c_{w}^{\alpha, v}$,

$$
\xi_{v} p_{\xi^{\alpha} / \xi_{v}}-\left(p_{\xi^{\alpha}}-\sum_{w \in \operatorname{supp} \alpha} b_{w}^{\alpha, v} p_{\xi^{\alpha-e_{w}}}+\sum_{\substack{|\beta|=2 \\ \beta \leq \alpha}} c_{\beta}^{\alpha, v} p_{\xi^{\alpha-\beta}}\right)
$$

is a polynomial of degree $n-2$, which is orthogonal to $\Pi_{n-2}$, and hence is zero. 
The equation (5.2) can easily be solved for $p_{\xi^{\alpha}}$, thereby giving a recursive three term recurrence for $p_{\xi^{\alpha}}$. By taking the convex combination of these with coefficients $\frac{\alpha_{v}}{|\alpha|}$ one obtains (4.4). By way of comparison, the only other recurrence that I am aware of is a nonrecursive three term recurrence in the Prorial orthonormal basis (cf [P57]) for Jacobi polynomials on the standard triangle, i.e.,

$$
P_{k}^{n}(x, y):=h_{k, n}^{-1}(1-x)^{k} P_{k}^{(\gamma, \beta)}\left(\frac{2 y}{1-x}-1\right) P_{n-k}^{(2 k+\beta+\gamma+1, \alpha)}(2 x-1), \quad 0 \leq k \leq n
$$

where $h_{k, n}$ are the normalising constants. The matrices which need to be (pseudo)inverted to obtain a recursive three term recurrence have a tridiagonal block form (see [DX01:86-88] for the explicit formulas).

\section{Appendix}

Lemma 6.1. Let $\lambda:=2|\alpha|+|\kappa|+d-3, \beta \geq \alpha$ and $|\beta|=2$. Then the constants $c_{\alpha}^{\beta}$ and $c_{\alpha}^{\beta, v}$ in Theorem 4.3 and Proposition 5.1

$$
\begin{gathered}
c_{\beta}^{\alpha}=-c(\alpha, \alpha-\beta)+\sum_{\substack{v \in V \\
\alpha_{v}-\beta_{v} \geq 1}} \frac{\alpha_{v}}{|\alpha|} c\left(\alpha-e_{v}, \alpha-e_{v}-\beta\right)+\sum_{\substack{v \in V \\
\beta_{v} \geq 1}} \frac{\alpha_{v}}{|\alpha|} c\left(\alpha-e_{v}, \alpha-\beta\right) b_{v}^{\alpha}, \\
c_{\beta}^{\alpha, v}=-c(\alpha, \alpha-\beta)+c\left(\alpha-e_{v}, \alpha-e_{v}-\beta\right)+\sum_{w \in \operatorname{supp} \beta} b_{w}^{\alpha, v} c\left(\alpha-e_{w}, \alpha-\beta\right),
\end{gathered}
$$

simplify for $\lambda \neq 1$ as follows

$$
\begin{gathered}
c_{\beta}^{\alpha}=\frac{(\kappa+1+\alpha-\beta)_{\beta}}{|\alpha| \lambda^{2}(\lambda-1)} \frac{(-\alpha)_{\beta}}{\beta !}\left\{\frac{2|\alpha|}{\lambda+1}-2+\sum_{v \in \operatorname{supp} \beta} \beta_{v} \frac{\lambda\left(\beta_{v}-1\right)+1-\alpha_{v}}{\kappa_{v}+\alpha_{v}}\right\}, \\
c_{\beta}^{\alpha, v}=\frac{(\kappa+1+\alpha-\beta)_{\beta}}{\lambda^{2}(\lambda-1)} \frac{(-\alpha)_{\beta}}{\beta !}\left\{\frac{2}{\lambda+1}-\frac{\beta_{v}}{\alpha_{v}}+\frac{\beta_{v}}{\alpha_{v}} \frac{\lambda\left(\beta_{v}-1\right)+1-\alpha_{v}}{\kappa_{v}+\alpha_{v}}\right\} .
\end{gathered}
$$

Proof: $\quad$ Suppose $\lambda \neq 1$. Recall $\beta \leq \alpha$ and $|\beta|=2$. It follows from (2.8) that

$$
\begin{gathered}
-c(\alpha, \alpha-\beta)=(\kappa+1+\alpha-\beta)_{\beta} \frac{(-\alpha)_{\beta}}{\beta !}\left\{\frac{-1}{(\lambda+1)(\lambda+2)}\right\}, \\
c\left(\alpha-e_{v}, \alpha-e_{v}-\beta\right)=(\kappa+1+\alpha-\beta)_{\beta} \frac{(-\alpha)_{\beta}}{\beta !}\left\{\frac{\left(\kappa_{v}+\alpha_{v}-\beta_{v}\right)\left(\alpha_{v}-\beta_{v}\right)}{\lambda(\lambda-1) \alpha_{v}\left(\kappa_{v}+\alpha_{v}\right)}\right\}, \\
c\left(\alpha-e_{v}, \alpha-\beta\right)=(\kappa+1+\alpha-\beta)_{\beta} \frac{(-\alpha)_{\beta}}{\beta !}\left\{\frac{-\beta_{v}}{\lambda \alpha_{v}\left(\kappa_{v}+\alpha_{v}\right)}\right\} .
\end{gathered}
$$


The factorisations of (6.7) and (6.8) were obtained from the identities

$$
\begin{gathered}
\alpha_{v}\left(-\alpha+e_{v}\right)_{\beta}=(-\alpha)_{\beta}\left(\alpha_{v}-\beta_{v}\right), \quad\left(\kappa+1+\alpha-e_{v}-\beta\right)_{\beta}=\frac{\left(\kappa_{v}+\alpha_{v}-\beta_{v}\right)}{\left(\kappa_{v}+\alpha_{v}\right)}(\kappa+1+\alpha-\beta)_{\beta}, \\
(\kappa+1+\alpha-\beta)_{\beta-e_{v}}=\frac{(\kappa+1+\alpha-\beta)_{\beta}}{\kappa_{v}+\alpha_{v}}, \quad\left(-\alpha+e_{v}\right)_{\beta-e_{v}}=\frac{(-\alpha)_{\beta}}{-\alpha_{v}}, \quad \frac{1}{\left(\beta-e_{v}\right) !}=\frac{\beta_{v}}{\beta !},
\end{gathered}
$$

when $v \in \operatorname{supp} \beta$, respectively, and follow by inspection otherwise. Let

$$
A:=(\kappa+1+\alpha-\beta)_{\beta} \frac{(-\alpha)_{\beta}}{\beta !} .
$$

Using (6.7), the first sum in (6.2) can be split

$$
\frac{A}{|\alpha| \lambda(\lambda-1)}\left\{\sum_{\substack{v \in V \\ \alpha_{v}-\beta_{v} \geq 1}}\left(\alpha_{v}-\beta_{v}\right)-\sum_{\substack{v \in V \\ \alpha_{v}-\beta_{v} \geq 1}} \frac{\beta_{v}\left(\alpha_{v}-\beta_{v}\right)}{\kappa_{v}+\alpha_{v}}\right\} .
$$

The restriction $\alpha_{v}-\beta_{v} \geq 1$ can be removed from both sums above, and so we obtain

$$
\frac{A}{|\alpha| \lambda(\lambda-1)}\left\{|\alpha|-2-\sum_{v \in \operatorname{supp} \beta} \frac{\beta_{v}\left(\alpha_{v}-\beta_{v}\right)}{\kappa_{v}+\alpha_{v}}\right\}
$$

Using (6.8) and (4.5) in the form

$$
b_{v}^{\alpha}=\left(\kappa_{v}+\alpha_{v}\right) \frac{2|\alpha|-\lambda-2}{\lambda(\lambda+2)}+\frac{1-\alpha_{v}}{\lambda}
$$

the second sum in (6.2) can be written

$$
\begin{aligned}
A \sum_{v \in \operatorname{supp} \beta}\left\{\beta_{v} \frac{\lambda+2-2|\alpha|}{|\alpha| \lambda^{2}(\lambda+2)}+\frac{\beta_{v}\left(\alpha_{v}-1\right)}{|\alpha| \lambda^{2}\left(\kappa_{v}+\alpha_{v}\right)}\right\} \\
=A\left\{2 \frac{\lambda+2-2|\alpha|}{|\alpha| \lambda^{2}(\lambda+2)}+\sum_{v \in \operatorname{supp} \beta} \frac{\beta_{v}\left(\alpha_{v}-1\right)}{|\alpha| \lambda^{2}\left(\kappa_{v}+\alpha_{v}\right)}\right\}
\end{aligned}
$$

Adding (6.6), (6.9) and (6.10), we get

$$
c_{\beta}^{\alpha}=A\left\{\frac{2(|\alpha|-\lambda-1)}{|\alpha| \lambda^{2}\left(\lambda^{2}-1\right)}+\frac{1}{|\alpha| \lambda^{2}(\lambda-1)} \sum_{v \in \operatorname{supp} \beta} \beta_{v} \frac{\lambda\left(\beta_{v}-1\right)+1-\alpha_{v}}{\kappa_{v}+\alpha_{v}}\right\},
$$

which gives (6.4).

Now we consider $c_{\beta}^{\alpha, v}$. We may write $(6.7)$ as

$$
c\left(\alpha-e_{v}, \alpha-e_{v}-\beta\right)=A\left\{\frac{1}{\lambda(\lambda-1)}+\frac{-\beta_{v}}{\lambda(\lambda-1) \alpha_{v}}+\frac{-\beta_{v}\left(\alpha_{v}-\beta_{v}\right)}{\lambda(\lambda-1) \alpha_{v}\left(\kappa_{v}+\alpha_{v}\right)}\right\}
$$


Since

$$
\left(\alpha_{w}-\delta_{v w}\right)\left(\kappa_{w}+\alpha_{w}-\delta_{v w}\right)=\alpha_{w}\left(\kappa_{w}+\alpha_{w}\right)-\delta_{v w}\left(\kappa_{w}+\alpha_{w}+\alpha_{w}-1\right)
$$

(5.5) can be written

$$
b_{w}^{\alpha, v}=\frac{2 \alpha_{w}\left(\kappa_{w}+\alpha_{w}\right)}{\lambda(\lambda+2)}-\delta_{v w}\left(\frac{\kappa_{w}+\alpha_{w}}{\lambda}+\frac{\alpha_{w}-1}{\lambda}\right) .
$$

Using this, and (6.8) with $v=w$, the sum in (6.3) becomes

$$
\begin{aligned}
& A \sum_{w \in \operatorname{supp} \beta}\left\{\frac{-2 \beta_{w}}{\lambda^{2}(\lambda+2)}+\right.\left.\delta_{v w}\left(\frac{\beta_{w}}{\lambda^{2} \alpha_{w}}+\frac{\beta_{w}\left(\alpha_{w}-1\right)}{\lambda^{2} \alpha_{w}\left(\kappa_{w}+\alpha_{w}\right)}\right)\right\} \\
&=A\left\{\frac{-4}{\lambda^{2}(\lambda+2)}+\frac{\beta_{v}}{\lambda^{2} \alpha_{v}}+\frac{\beta_{v}\left(\alpha_{v}-1\right)}{\lambda^{2} \alpha_{v}\left(\kappa_{v}+\alpha_{v}\right)}\right\} .
\end{aligned}
$$

Finally, adding (6.6), (6.11) and the above gives

$$
c_{\beta}^{\alpha, v}=A\left\{\frac{2}{\lambda^{2}\left(\lambda^{2}-1\right)}+\frac{-\beta_{v}}{\lambda^{2}(\lambda-1) \alpha_{v}}+\beta_{v} \frac{\lambda\left(\beta_{v}-1\right)+1-\alpha_{v}}{\lambda^{2}(\lambda-1) \alpha_{v}\left(\kappa_{v}+\alpha_{v}\right)}\right\}
$$

which is $(6.5)$.

\section{Acknowledgement}

Thanks to Nira Dyn for her encouragement with this work.

\section{References}

[AK26] P. Appell and J. Kampé de Fériet, "Fonctions Hypergéométriqes et Hypersphériques

- Polynomes d'Hermite", Gauthier-Villars, Paris, 1926.

[BPS10] R. Barrio, J. M. Peña, and T. Sauer, Three term recurrence for the evaluation of multivariate orthogonal polynomials, J. Approx. Theory 162 (2010), 407-420.

[BS92] H. Berens and H. J. Schmid, On the number of nodes of odd degree cubature formulae for integrals with Jacobi weights on a simplex, in "Numerical integration, NATO Adv. Sci. Inst. Ser. C Math. Phys. Sci., 357" (T. O. Espelid and A. Genz Eds.), pp. 37-44, Kluwer Acad. Publ., Dordrecht, 1992.

[B87] C. de Boor, $B$-form basics, in "Geometric Modeling: Algorithms and New Trends" (G. E. Farin Ed.), pp. 131-148, SIAM Publications, Philadelphia, 1987.

[B83] L. P. Bos, Bounding the Lebesgue function for Lagrange interpolation in a simplex, J. Approx. Theory 38 (1983), 43-59.

[CW02] S. Cooper and S. Waldron, The diagonalisation of the multivariate Bernstein operator, J. Approx. Theory 117 (2002), 103-131. 
[D91] M. Dubiner, Spectral methods on triangles and other domains, J. Sci. Comput. 6 (1991), 345-390.

[DX01] C. F. Dunkl and Yuan Xu, "Orthogonal polynomials of several variables", Cambridge University Press, Cambridge, 2001.

[EMOT53] A. Erdélyi, W. Magnus, F. Oberhettinger, and F. Tricomi, "Higher transcendental functions (three volumes).", McGraw-Hill Book Company, Inc, New York, 1953-55.

[IM91] M. Ismail and D. R. Masson, Two families of orthogonal polynomials related to Jacobi polynomials, Rocky Mountain J. Math. 21 (1991), 359-375.

[K82a] M. A. Kowalski, The recursion formulas for orthogonal polynomials in $n$ variables, SIAM J. Math. Anal. 13 (1982), 309-315.

[K82b] M. A. Kowalski, Orthogonality and recursion formulas for polynomials in $n$ variables, SIAM J. Math. Anal. 13 (1982), 316-323.

[P57] J Proriol, Sur une famille de polynomes à variables orthogonaux dans un triangle, $C$. R. Acad. Sci. Paris 245 (1957), 2459-2461.

[S99] P. K. Suetin, "Orthogonal polynomials in two variables (translation from Russian)", Analytical Methods and Special Functions Volume 3, Gordon and Breach Science Publishers, Amsterdam, 1999.

[W06] S. Waldron, On the Bernstein-Bézier form of Jacobi polynomials on a simplex, $J$. Approx. Theory 140 (2006), 86-99.

[X93] Yuan. Xu, On multivariate orthogonal polynomials, SIAM J. Math. Anal. 24 (1993), 783-784.

[X05] Yuan Xu, Monomial orthogonal polynomials of several variables, J. Approx. Theory 133 (2005), 1-37.

[X10] Yuan Xu, Fourier series and approximation on hexagonal and triangular domains, Constr. Approx. 31 (2010), 115-138. 